\title{
Le Corpus of Anglo-Saxon Stone Sculpture in England: Constitution, documentation numérique et enjeux
}

The Corpus of Anglo-Saxon Stone Sculpture in England: putting it together, digitally documenting it and envisaging what the future holds

\section{Celia Orsini et Sarah Semple}

\section{OpenEdition}

\section{Journals}

Édition électronique

URL : https://journals.openedition.org/cel/18284

DOI : $10.4000 /$ cel. 18284

ISSN : 2262-208X

Éditeur

École du Louvre

Référence électronique

Celia Orsini et Sarah Semple, "Le Corpus of Anglo-Saxon Stone Sculpture in England : Constitution, documentation numérique et enjeux », Les Cahiers de l'École du Louvre [En ligne], 17 | 2021, mis en ligne le 16 novembre 2021, consulté le 13 décembre 2021. URL : http://journals.openedition.org/cel/18284 ; DOI : https://doi.org/10.4000/cel.18284

Ce document a été généré automatiquement le 13 décembre 2021.

\section{(i)

Les Cahiers de l'École du Louvre sont mis à disposition selon les termes de la licence Creative Commons Attribution - Pas d'Utilisation Commerciale - Pas de Modification 4.0 International. 


\title{
Le Corpus of Anglo-Saxon Stone Sculpture in England : Constitution, documentation numérique et enjeux
}

The Corpus of Anglo-Saxon Stone Sculpture in England: putting it together, digitally documenting it and envisaging what the future holds

\author{
Celia Orsini et Sarah Semple
}

1 Le projet Corpus of Anglo-Saxon Stone Sculpture (CASSS) dirigé par Rosemary Cramp depuis 1977 a été soutenu par la British Academy, les Arts and Humanities Research Council et bien d'autres au cours de sa longue existence. Sa mission : identifier, documenter et publier sous la forme d'enregistrements standardisés toute la sculpture anglaise datée entre le $\mathrm{VII}^{\mathrm{e}}$ et le XII ${ }^{\mathrm{e}}$ siècle. Débuté à la fin des années 1970, le projet verra ses dernières publications paraître en 2022, totalisant plus de 40 ans de recherche. Parallèlement, le projet Worked in Stone (WIST), dernière phase du projet CASSS dont les inventaires couvrent l'ensemble de l'Angleterre, concrétisera les objectifs fixés au tout début de cette aventure sculpturale, en mettant en ligne en libre accès tous les catalogues, la documentation photographique et les ressources accumulées depuis les années 1970. Toutes les informations collectées seront ainsi à disposition du grand public dans la banque de données Archaeology Data Service (ADS) ${ }^{1}$.

\section{Dépasser l'inventaire, protéger le passé}

2 Les monuments sculptés forment un témoignage unique des transformations religieuses et politiques du haut Moyen Âge. Ils constituent souvent à eux seuls les premières traces visibles d'activités chrétiennes, indices précieux menant parfois à la fouille du site même. La réintroduction du christianisme aux $\mathrm{VI}^{\mathrm{e}}-\mathrm{VII}{ }^{\mathrm{e}}$ siècles dans le monde anglo-saxon amène des changements significatifs dans l'organisation de l'espace, avec la création de nouveaux habitats, de nouveaux sites funéraires et, dans le monde social et religieux, de nouvelles hiérarchies, de nouveaux contacts avec le 
continent, de nouvelles pratiques religieuses et funéraires, sociales et artistiques. Des hautes croix de Northumbrie au $\mathrm{VII}^{\mathrm{e}}$ siècle $^{2}$ aux pierres tombales anglo-scandinaves d'East Anglia, la pierre est un nouveau véhicule artistique (fig. 1), utilisé pour créer de puissants supports visuels et soutenir de nouvelles pratiques de commémoration ${ }^{3}$. Mais au-delà du fait de manifester l'impact des transformations religieuses du haut Moyen Âge, les sculptures anglo-saxonnes persistent dans le temps et beaucoup d'entre elles ont une vie qui dépasse leur fonction de monuments chrétiens ${ }^{4}$. Abandonnée, partiellement détruite, la pierre est aussi réutilisée en tant que matière première dans la construction, intégrée dans la fabrique des bâtiments, intentionnellement exposée ou pas 5 .

Fig. 1. Haute croix d'Aycliffe

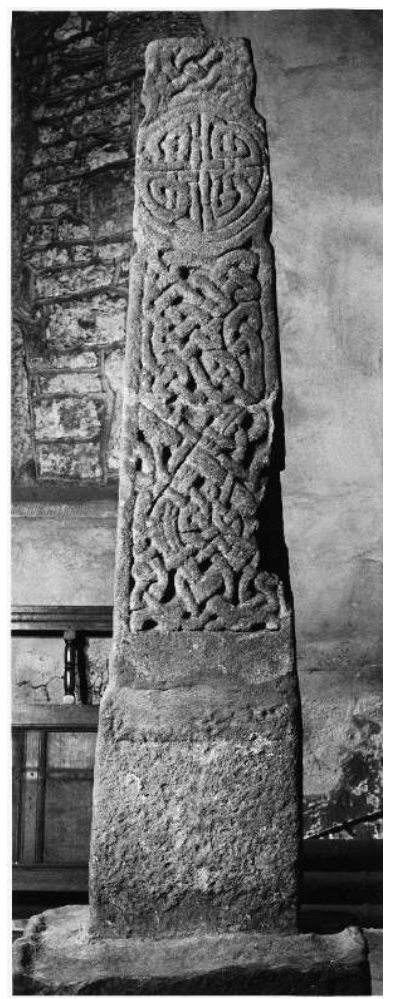

Haute croix d'Aycliffe 2C, dont les trois fragments ont été remontés (h. $143.5 \mathrm{~cm}, \mathrm{~L} 38 \mathrm{~cm}$ ), publiée dans Cramp, R. J. (1984), Corpus of Anglo-Saxon Stone Sculpture, 1: County Durham and Northumberland (Oxford University Press). Oxford.

(C) Photographie de Tom Middlemass / Cramp, R. J.

Dans ce contexte d'extrême diversité est né le Corpus of Anglo-Saxon Stone Sculpture, sous la tutelle de Rosemary Cramp, qui dépoussiéra avec ses chercheurs les recoins oubliés d'églises et de cimetières. La fonction du corpus depuis ses débuts est d'identifier et d'enregistrer chaque fragment sculpté du $\mathrm{VII}^{\mathrm{e}}$ au XII ${ }^{\mathrm{e}}$ siècle. L'équipe du corpus a établi un format d'enregistrement précis, rigoureusement appliqué depuis les années 1980 jusqu'à nos jours. L'approche de départ est simple ; il s'agit de réaliser et de publier un inventaire exhaustif par counties. La taille de ces divisions administratives est très variable et souvent les inventaires de plusieurs counties sont regroupés par région pour la publication. Le corpus fournit un compte rendu précis et détaillé de chaque monument, un enregistrement photographique mais aussi dans la mesure du possible une interprétation du contexte archéologique, historique et paysager. 
4 Dès le début $\mathrm{du} \mathrm{XX}^{\mathrm{e}}$ siècle, on retrouve la sculpture anglo-saxonne sous de multiples formes; de toutes les tailles et qu'il s'agisse de fragments ou de monuments préservés in situ, ceux-ci sont encore souvent mal évalués et restent incompris par leur public. Parfois, ils sont plus ou moins bien protégés dans les églises ou les cimetières, intégrés à la maçonnerie ou non, visibles sur un côté, ou cachés dans les fondations des bâtiments, conservés dans des collections privées ou dans des musées. Ces sculptures au statut ambivalent, à la fois œuvres d'art et artefacts archéologiques, sont rarement mises en valeur, souvent elles sont empilées dans un recoin oublié, dans une crypte, ou pleinement exposées aux intempéries. On distingue peut-être les bonnes intentions sous-jacentes derrière ces regroupements mais le manque de compréhension globale de la valeur des objets reste flagrant. Depuis ses débuts, CASSS s'entête et s'efforce de stimuler les recherches autour de la sculpture anglaise et de diffuser ces nouveaux acquis de connaissances auprès du plus grand nombre dans le but d'améliorer la prise en charge et la protection de ces vestiges.

\section{Un parcours décisionnel adroit}

5 Ce projet gigantesque a été monté par le Professeur Dame Rosemary Cramp en 1972. Elle partage dans le premier volume de la série sa vision du Corpus. Les premiers choix sont difficiles mais déterminants pour le futur de CASSS. R. Cramp s'impose un cadre géographique limité à l'Angleterre ${ }^{6}$, vaste entité politique aux limites géographiques strictes, et un cadre chronologique spécifique, englobant la christianisation des royaumes anglo-saxons jusqu'aux conquêtes normandes. Des problèmes sémantiques découlent inévitablement de la création d'un inventaire précis délimité dans le temps et l'espace et les terminologies peuvent encore aujourd'hui faire débat. Pourtant, en publiant son volume fondateur en 1984, R. Cramp établit la grammaire de l'iconographie de la sculpture anglo-saxonne et détermina un vocabulaire commun évoluant au fil des découvertes mais demeurant néanmoins le fondement sur lequel toutes les études de la sculpture anglaise ont été fondées depuis ${ }^{7}$. Ce système fut tout d'abord publié en introduction du premier volume de la série, puis séparément en 19918. R. Cramp y détermine un système descriptif standardisé et homogène, qui comprend le lieu de découverte, le type, la forme et l'origine du monument, ses dimensions, son iconographie, son niveau de préservation, et deux sections descriptives et interprétatives. Dans ces dernières, une attention particulière est portée à l'établissement d'une datation argumentée; elles sont suivies d'une compilation exhaustive de références bibliographiques se rapportant au monument.

6 La collecte et l'analyse des données passent par des recherches historiographiques ardues et un travail de terrain qui se veut aussi complet que possible, revisitant les travaux d'antiquaires et répertoriant les nouvelles découvertes sur l'ensemble d'une région. Cela permet également de replacer toutes les œuvres dans leur contexte historique et de réévaluer leur emplacement actuel. Grâce à l'établissement de la «Grammaire » du corpus, l'identification des monuments par type (stèle ou couverture de tombe, élément architectural, hogback, etc.) précède l'analyse des types d'ornementation et d'entrelacs (rinceau de vigne, ornement végétal ou animal, iconographie figurée de personnages saints ou séculiers) et des inscriptions (runiques ou en lettres romaines, en latin ou en vieil anglais). 
7 Après près de cinquante ans d'existence du projet, nous en sommes actuellement au $13^{\text {e }}$ volume publié, ce qui recouvre au total plus de 30 counties d'Angleterre (fig. 2). La longévité du projet est en soi un hommage à Rosemary Cramp, qui a dû batailler année après année pour maintenir le projet à flot et obtenir le financement de ses recherches. Il reste trois volumes à paraître sous sa direction, impliquant la participation et l'engagement entre autres de treize auteurs, dix chercheurs et spécialistes, de multiples géologues, et de photographes. Tous se sont engagés sur le long terme à produire des catalogues d'excellente qualité tout en conservant un modèle uniforme facilitant leur consultation et leur utilisation par tous les futurs chercheurs.

Fig. 2. Carte d'Angleterre illustrant les counties qui ont fait l'objet d'une publication dans la série Corpus of Anglo-Saxon Stone Sculpture

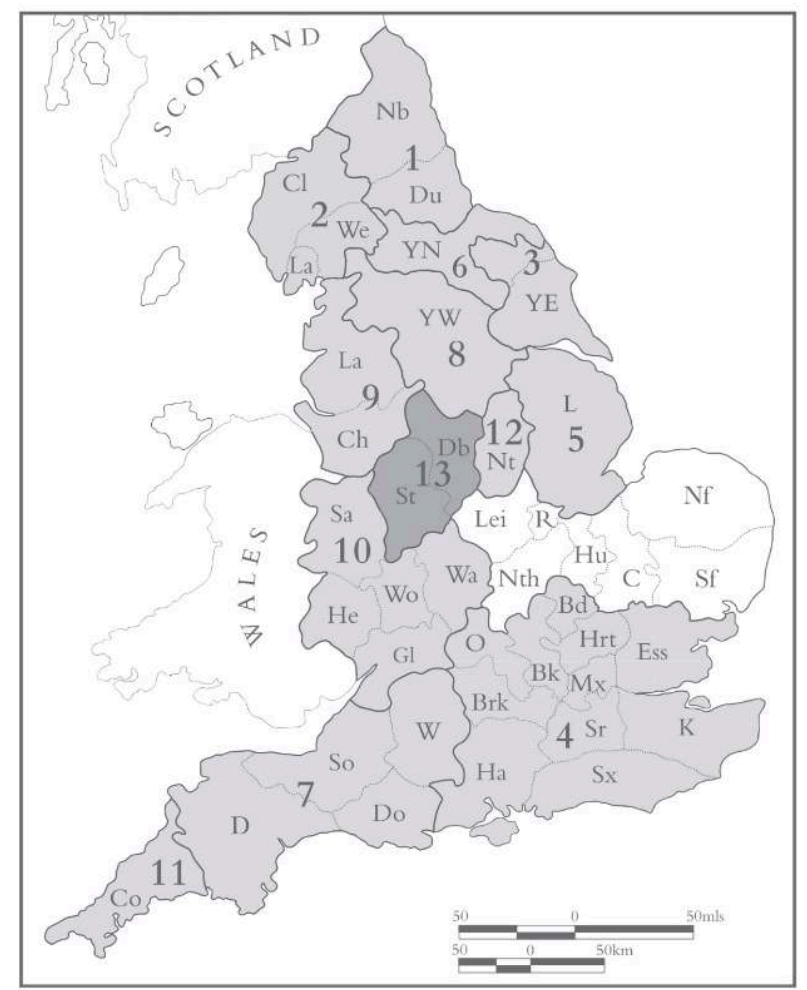

Carte d'Angleterre illustrant les counties qui ont fait l'objet d'une publication dans la série Corpus of Anglo-Saxon Stone Sculpture en gris et les counties dont les publications sont en cours de préparation en blanc. Dessinée par Yvonne Beadnell, publiée dans Hawkes, J. et Sidebottom, P. (2018), Corpus of Anglo-Saxon Stone Sculpture, 13: Derbyshire and Staffordshire (Oxford University Press). Oxford.

(C) Rosemary Cramp

8 Chacune de ces publications s'attache à produire des analyses informées des monuments sculptés, mais plusieurs chapitres s'intéressent plus spécifiquement aux formes et motifs développés dans chaque région. L'établissement du corpus permet la mise en évidence de différences régionales très marquées alors même que l'on observe un ensemble de monuments de même type ce qui permet parfois de mettre en évidence des ateliers de production locaux ou centraux ${ }^{9}$. Grâce aux contributions de spécialistes, on notera l'importance donnée aux matériaux, à l'étude de leur provenance proche ou lointaine et aux techniques de taille utilisées qui forment les premiers indicateurs d'ateliers (voir à Jarrow et à Wearmouth ${ }^{10}$ ). L'identification de la source des pierres sculptées permet de s'interroger sur la réutilisation des matériaux romains, 
l'extraction de la pierre et la localisation des carrières, mais également sur le transport des matériaux. Les chapitres introductifs qui précèdent les catalogues de l'inventaire tentent de rassembler un maximum de preuves pour donner autant de sens aux grands monuments in situ qu'aux fragments isolés. Un chapitre est dédié au contexte historique de chaque county complété par l'historiographie de la région, dont la richesse peut varier d'un extrême à l'autre ; ce lien renseigne parfois explicitement sur la richesse sculpturale actuelle de certaines régions par rapport à d'autres ${ }^{11}$.

\section{Les nouveaux enjeux du passage au numérique}

En 2006, de nouvelles thématiques ont fait leur chemin au sein de l'équipe. Comment rendre plus accessible cet inventaire faramineux et ces sources d'informations, tant aux chercheurs qu'au grand public ? En harmonie avec les technologies des années 2000, on s'interrogea sur la traduction des catalogues en une base de données en ligne. Il fallut attendre plusieurs années pour que cela soit véritablement possible. Le projet se dota d'un site internet ${ }^{12}$, tenta de construire une première base de données en ligne et entra courageusement dans une nouvelle ère informatique (fig. 3). Les mises en ligne se firent par volume. La rapidité avec laquelle le projet progressa est directement liée au développement de la reconnaissance optique de texte (OCR). Enfin, les archives volumineuses purent également être scannées et permirent la mise en ligne de l'information, non seulement textuelle mais également photographique. Alors que le projet poursuivait ses recherches pour étendre sa couverture géographique, la base de données en ligne se construisait lentement au fil des subventions sporadiques, pendant près de dix ans.

Fig. 3. Page d'accueil du site internet du Corpus of Anglo-Saxon Stone Sculpture
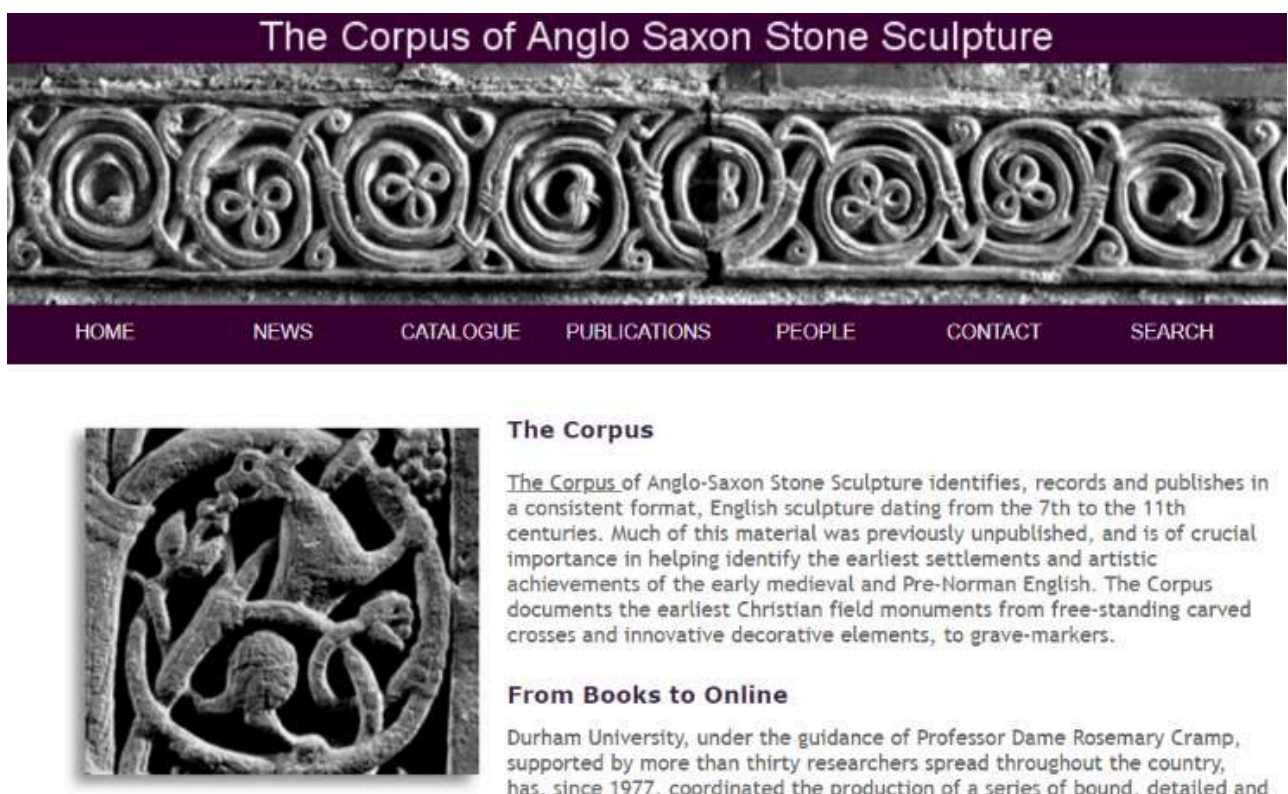

The Corpus

The Corpus of Anglo-Saxon Stone Sculpture identifies, records and publishes in a consistent format, English sculpture dating from the 7 th to the 11 th centuries. Much of this material was previously unpublished, and is of crucial importance in helping identify the earliest settlements and artistic achievements of the early medieval and Pre-Norman English. The Corpus documents the earliest Christian field monuments from free-standing carved crosses and innovative decorative elements, to grave-markers.

From Books to Online

Durham University, under the guidance of Professor Dame Rosemary Cramp, supported by more than thirty researchers spread throughout the country, has, since 1977, coordinated the production of a series of bound, detailed and fully fllustrated volumes that provide coverage of every early medieval Sculpture in England. In recent years, with the support of the AHRC, British

Accessible à l'adresse http://www.ascorpus.ac.uk/

En 2017, les Professeurs Cramp et Semple parvinrent à obtenir un financement conséquent de l'AHRC et lancèrent le projet Worked in Stone. WIST constitue la dernière 
phase du projet CASSS. Pour ce faire, les Professeurs Cramp et Semple de l'université de Durham s'associent aux Professeurs Jane Hawkes et Julian Richard de l'Université de York, à Joanna Story de l'université de Leicester et à Helen Gittos de l'université d'Oxford. Quatre chercheurs (Derek Craig, Belinda Burke, Tudor Skinner et Celia Orsini) viennent compléter l'équipe de recherche à Durham, alors que le projet continue de s'associer à de nombreux autres acteurs, notamment Dominique Powlesland pour le développement de nouvelles restitutions graphiques et Sarah Price pour la construction des premiers systèmes numériques et la gestion de l'hébergement du site internet.

11 Le projet Worked In Stone s'est donné entre autres pour mission :

12 de mener à terme les recherches lancées dans les années 1980. C'est-à-dire de continuer et de terminer l'inventaire des sculptures anglo-saxonnes en Angleterre par la production des trois derniers volumes du Corpus, à savoir: Leicestershire, Cambridgeshire et East Anglia ;

13 de développer l'impact du Corpus et la place de la sculpture anglo-saxonne dans les recherches archéologiques actuelles. Pour cela, trois journées d'étude ont été organisées pour explorer la place de la sculpture dans l'univers des spécialistes et nonspécialistes (par exemple le travail sur les sculptures dans les écoles, les musées et les églises rurales). Ces journées ont également servi à identifier des problèmes de conservation, de mise en valeur et de protection physique et légale de ces objets dans certains lieux et ont permis de s'interroger sur les moyens de diffusion des connaissances archéologiques ;

de créer une nouvelle base de données et un moteur de recherche libre d'accès en travaillant avec ADS. Ce dernier point est particulièrement important puisque c'est par là que nous allons tenter de diffuser l'information recueillie à un maximum de personnes.

\section{La dématérialisation des archives}

Le passage de la documentation physique au numérique a été possible grâce aux avancées de la technologie $O C R$, la reconnaissance optique des caractères. Les premiers volumes n'avaient bien sûr pas de version numérique permettant le transfert des données du livre dans une base de données de type Excel, les premières tentatives de catalogues en ligne ont donc inclus un traitement à rebours passant du livre numérisé vers la création d'un inventaire numérique; les archives photographiques ont été traitées de la même façon. Après le traitement $O C R$, chaque entrée des catalogues a été manuellement corrigée et formatée. Avec les avancées technologiques progressives, l'amélioration des OCR, la mise à disposition de textes et de photographies déjà numériques, le traitement des données s'est fait plus rapide et a permis la mise en ligne d'archives de meilleure qualité (fig. 4 et 5). À partir de 2018, les financements obtenus par le projet WIST ont permis l'accélération des mises à jour de la base de données. La généralisation des bases de données en ligne amène l'équipe de recherche à s'interroger sur la nécessité de protéger les données afin de conserver leur intégrité dans le temps en garantissant leur accessibilité à perpétuité. En s'associant à la Plateforme Archaeology Data Services ${ }^{13}$, actuellement basée à l'université de York, WIST s'engage à fournir une base de données qui sera accessible à titre gratuit sur le très long terme d'ici la fin 2022, et un nouveau moteur de recherche sera mis en place afin de 
faciliter le croisement des données de CASSS. Toutes les entrées des catalogues publiés par le corpus seront disponibles en ligne et les enregistrements photographiques téléchargeables par tout utilisateur. Afin d'utiliser ce moteur de recherche, CASSS a dû adopter de nouvelles normes et créer tout un système de métadonnées qui permettront à l'utilisateur de faire des recherches par mots-clefs, par types de monuments ou de motifs, par régions, par ensembles chronologiques, etc., mais surtout de croiser ces données et d'obtenir des groupes et des sous-groupes selon des critères géographiques et géologiques et d'en retirer des cartes archéologiques. Au niveau numérique, le projet Worked in Stone commence à voir la lumière au bout du tunnel. Reste cependant le problème de la mise à jour et de l'actualisation de l'inventaire CASSS, que l'on se soucie de sa production en support papier ou de la mise à jour de ses bases de données numériques. Actuellement, CASSS ne peut pas s'assurer de la publication des mises à jour d'inventaires, sachant pourtant que certains des catalogues remontent aux années 1980 et nécessiteraient des compléments de publication contenant les nouvelles découvertes réalisées. À la différence du corpus SATHMA couplé à la base CARE-France qui se veut évolutive et permet la mise à jour permanente des inventaires ${ }^{14}$, CASSS devra mobiliser de nouveaux financements pour pouvoir modifier et renouveler l'information archéologique sculpturale.

Fig. 4. Colonne sculptée de croix dressée de Derby

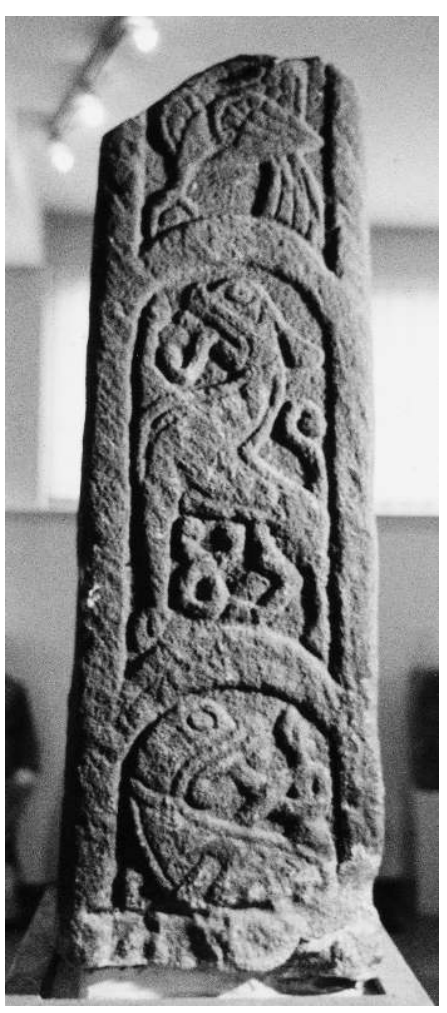

Colonne sculptée de croix dressée de Derby $1(\mathrm{H} .75 \mathrm{~cm}, \mathrm{~L} .37 \mathrm{~cm})$, publiée dans Hawkes, J. et Sidebottom, P. (2018), Corpus of Anglo-Saxon Stone Sculpture, 13 : Derbyshire and Staffordshire (Oxford University Press). Oxford.

(c) Photographie de J. Hawkes et P.C. Sidebottom / Hawkes, J. et Sidebottom 
Figure 5. Colonne sculptée de Brailsford

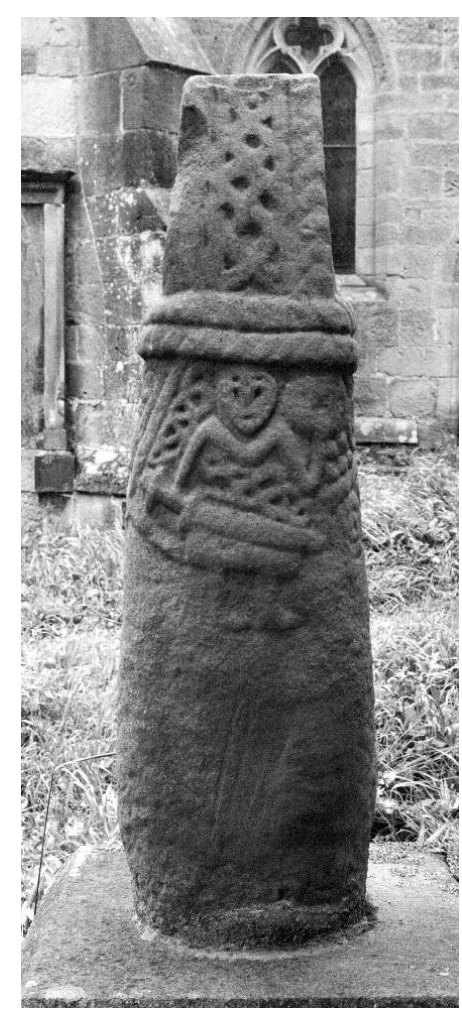

Colonne sculptée de Brailsford 1 (H. 132cm, L. 40cm), publiée dans Hawkes, J. et Sidebottom, P. (2018), Corpus of Anglo-Saxon Stone Sculpture, 13 : Derbyshire and Staffordshire (Oxford University Press). Oxford.

(c) Photographie de J. Hawkes et P.C. Sidebottom / Hawkes, J. et Sidebottom

\section{Le lien passé-présent}

Pour l'instant, les treize volumes parus sont mis en ligne à disposition des utilisateurs sur le site internet [ascorpus.ac.uk] et recensent plus de 2500 fragments sculptés et plus de 10000 images. Ces trois dernières années de travail ont permis de compléter l'approche générale de CASSS avec l'utilisation de la photogrammétrie afin de créer des modèles 3D. Ces modèles permettent une analyse iconographique en profondeur, ils aident à la compréhension de certains reliefs érodés, ainsi qu'à clarifier certaines inscriptions, jusqu'à présent trop altérées pour en comprendre la signification; ils ont aussi permis de repérer des traces de peintures jusque-là non identifiées. C'est un outil nouveau pour le corpus qui se révèle être particulièrement intéressant et promet d'apporter des informations inédites sur des monuments déjà connus. Fort de ce nouvel aspect technologique, WIST ajoute de nouvelles flèches à son arc: une forte présence sur les réseaux sociaux, notamment Facebook et Twitter, a permis de populariser les données déjà acquises et de constamment promouvoir les nouvelles de l'archéologie de la sculpture anglaise. Une conférence internationale, la publication de ces actes mais surtout d'un ouvrage de synthèse d'ici la fin 2022, célèbreront l'achèvement du projet Corpus of Anglo-Saxon Stone Sculpture, faisant un retour attendu sur ses réussites passées, les difficultés rencontrées en chemin et ses espoirs concernant la revitalisation de ce sujet d'étude ${ }^{15}$. L'objectif de ces derniers événements est de ramener sur le devant de la scène la sculpture anglo-saxonne et de poursuivre ce qui a toujours été l'objectif 
premier du projet CASSS : l'émulation des recherches concernant la sculpture anglosaxonne et la diffusion des connaissances au plus grand nombre pour une meilleure compréhension et une meilleure gestion de ces vestiges du passé.

\section{NOTES}

1. ADS est une organisation à but non lucratif, spécialisée dans la préservation de toute donnée numérique reliée à la conservation du patrimoine national. L'objectif principal d'ADS est de rassembler, cataloguer et préserver toute ressource numérique créée dans le contexte des recherches archéologiques. Cette organisation permet également de promouvoir des normes standards de gestion de données informatiques archéologiques. https:// archaeologydataservice.ac.uk/

2. Jane Hawkes, « Northumbrian Sculpture: Questions of Context », Northumbria's golden age, sous la direction de Jane Hawkes et Susan Mills, Sutton, University of Michigan, 1999, pp. 204-215.

3. John Blair, The church in Anglo-Saxon Society, Oxford, Oxford University Press, 2005.

4. Howard Williams, Joanne Kirton et Meggen Gondeck, «Introduction: Stones in Substance, Space and Time ", Early Medieval Stone Monuments, Woodbridge, Boydell Press, 2015, pp. 1-34.

5. Sam Turner, Sarah Semple et Alex Turner, One monastery in two places: Wearmouth and Jarrow past and present, Hatfield, University of Hertfordshire Press, 2013.

6. D'autres ouvrages viendront s'ajouter aux inventaires consacrés à la sculpture anglo-saxonne, notamment celui de Derek Craig, The distribution of Pre-Norman sculpture in South-west Scotland: provenance, ornament and regional groups, Durham, Thèse Durham University, 1992.

7. Rosemary Cramp, Corpus of Anglo-Saxon Stone Sculpture, 1: County Durham and Northumberland, Oxford, Oxford University Press, 1984.

8. R. Cramp, Grammar of Anglo-Saxon Ornament, Oxford, Oxford University Press, 1991.

9. Cramp parle de Schools of carving notamment dans le nord de la Grande-Bretagne. R. Cramp, op. cit. note 7 .

10. R. Cramp, Wearmouth and Jarrow monastic sites, Swindon, English Heritage, 2005.

11. Il est intéressant d'observer ces effets historiographiques sur l'établissement des inventaires entre le Nottinghamshire et le North Yorkshire par exemple. James Lang, Corpus of Anglo-Saxon Stone Sculpture, 6: North Yorkshire, Oxford, Oxford University Press, 2006 ; Paul Everson et David Stocker, Corpus of Anglo-Saxon Stone Sculpture, 12: Nottinghamshire, Oxford, Oxford University Press, 2016.

12. http://www.ascorpus.ac.uk/

13. https://archaeologydataservice.ac.uk/

14. Pascale Chevalier, Jessy Crochat, Anne Flammin, Pierre-Yves Le Pogam, Anne-Bénédicte Mérel-Brandenburg, Arnaud Millereux et Christian Sapin, «Le Projet Care-France : dernières avancées et extension de la base de données en ligne au domaine de la sculpture des $\mathrm{IV}^{\mathrm{e}}-\mathrm{X}^{\mathrm{e}}$ siècles ", Hortus Artium Medievalium, 24, 2018, p. 8-13.

15. L'organisation de la conférence "Worked in Stone: Early Medieval Sculpture in its International Context » et la publication de ses actes se tiendront à Durham en 2022, date révisée si nécessaire en fonction de la situation sanitaire actuelle. 


\section{RÉSUMÉS}

Après près d'un demi-siècle de recherche, le projet Corpus of Anglo-Saxon Stone Sculpture (CASSS), coordonné par le Professeur Rosemary Cramp touche à sa fin. Il regroupe les inventaires de tous les restes sculpturaux de type anglo-saxon, datés entre le $\mathrm{VII}^{\mathrm{e}}$ et le $\mathrm{XII}^{\mathrm{e}}$ siècle. Avec les publications finales en 2022, il regroupera plus de dix-huit publications dont seize consacrées aux inventaires de chaque counties d'Angleterre. La fin de ce projet colossal est l'occasion de revenir sur les atouts et les limites d'un projet construit sur le long terme qui a vécu et survécu aux grandes transformations apportées par l'ère du numérique.

After almost half a century of research, the Corpus of Anglo-Saxon Stone Sculpture (CASSS) project, coordinated by Professor Rosemary Cramp, is coming to an end. It brings together inventories of all Anglo-Saxon sculptural remains dating from between the seventh and twelfth centuries. With the final publications in 2022, it will comprise over eighteen publications, sixteen of which are devoted to inventories from each county in England. The end of this colossal project is an opportunity to look back at the strengths and limitations of an operation built over the long term that has survived the great transformations brought about by the digital age.

\section{INDEX}

Keywords : Corpus of Anglo-Saxon Stone Sculpture, Britain, sculpture, seventh-twelfth century Mots-clés : Corpus Anglo-Saxon Stone Sculpture, Angleterre, sculpture, VIIe-XIIe siècle

\section{AUTEURS}

\section{CELIA ORSINI}

Celia Orsini est actuellement chercheur indépendante, associée au projet Worked in Stone: Completing the Corpus of Anglo-Saxon Stone Sculpture de l'Université de Durham. Ancienne élève de l'université Paris I Panthéon-Sorbonne, elle a soutenu, en 2017, une thèse de doctorat en archéologie sur les héritages monumentaux utilisés dans la construction des paysages funéraires dans la région Tyne-Forth entre le $\mathrm{V}^{\mathrm{e}}$ et le $\mathrm{VIII}^{\mathrm{e}}$ siècle. Elle est devenue chercheur postdoctorante à Durham intégrant l'équipe de WIST en 2018. Elle se consacre actuellement à l'étude des pratiques funéraires du haut Moyen Âge en Europe et cultive un intérêt tout particulier pour le développement de la médecine au haut Moyen Âge.

Celia Orsini is currently an independent researcher involved in the project Worked in Stone: Completing the Corpus of Anglo-Saxon Stone Sculpture at Durham University. A graduate of the Université Paris I Panthéon-Sorbonne, she defended her doctoral thesis in archaeology on the monumental legacies used in the construction of funerary landscapes in the Tyne-Forth region (fifth-eighth centuries) in 2017. She became a post-doctoral researcher at Durham, joining the WIST team in 2018. She is currently studying funerary practices in early medieval Europe and is particularly interested in the development of medicine during the Early Middle Ages.

\section{SARAH SEMPLE}

Sarah Semple est professeur d'archéologie médiévale à l'université de Durham. Après avoir été étudiante à l'Institute of Archaeology à Londres, elle a soutenu sa thèse de Doctorat à l'université 
d'Oxford. Elle travaille à l'université de Durham depuis 2006 en tant qu'archéologue spécialiste du haut Moyen Âge avec un attrait pour la culture matérielle de la Grande-Bretagne et de l'Europe du Nord. Elle mène à bien plusieurs projets de renommée internationale dont The assembly Project, Wearmouth and Jarrow, Yeavering : A Palace in its Landscape, et Worked in Stone : Completing the Corpus of Anglo-Saxon Stone Sculpture.

Sarah Semple is professor of medieval archaeology at Durham University. After her studies at the Institute of Archaeology in London, she defended her doctoral thesis at Oxford University. She has been working at Durham University since 2006 as an archaeologist specialising in the Early Middle Ages with a focus on the material culture of Britain and Northern Europe. She has participated in several internationally renowned projects including The Assembly Project, Wearmouth and Jarrow, Yeavering: A Palace in its Landscape, and Worked in Stone: Completing the Corpus of Anglo-Saxon Stone Sculpture. 